Document downloaded from:

http://hdl.handle.net/10251/118391

This paper must be cited as:

Barbi, M.; Garcia-Pardo, C.; Cardona Marcet, N.; Andrea Nevárez; Vicente Pons Beltrán; Frasson, M. (2018). Impact of Receivers Location on the Accuracy of Capsule Endoscope Localization. En 2018 IEEE 29th Annual International Symposium on Personal, Indoor and Mobile Radio Communications (PIMRC). IEEE. 340-344.

https://doi.org/10.1109/PIMRC.2018.8580862

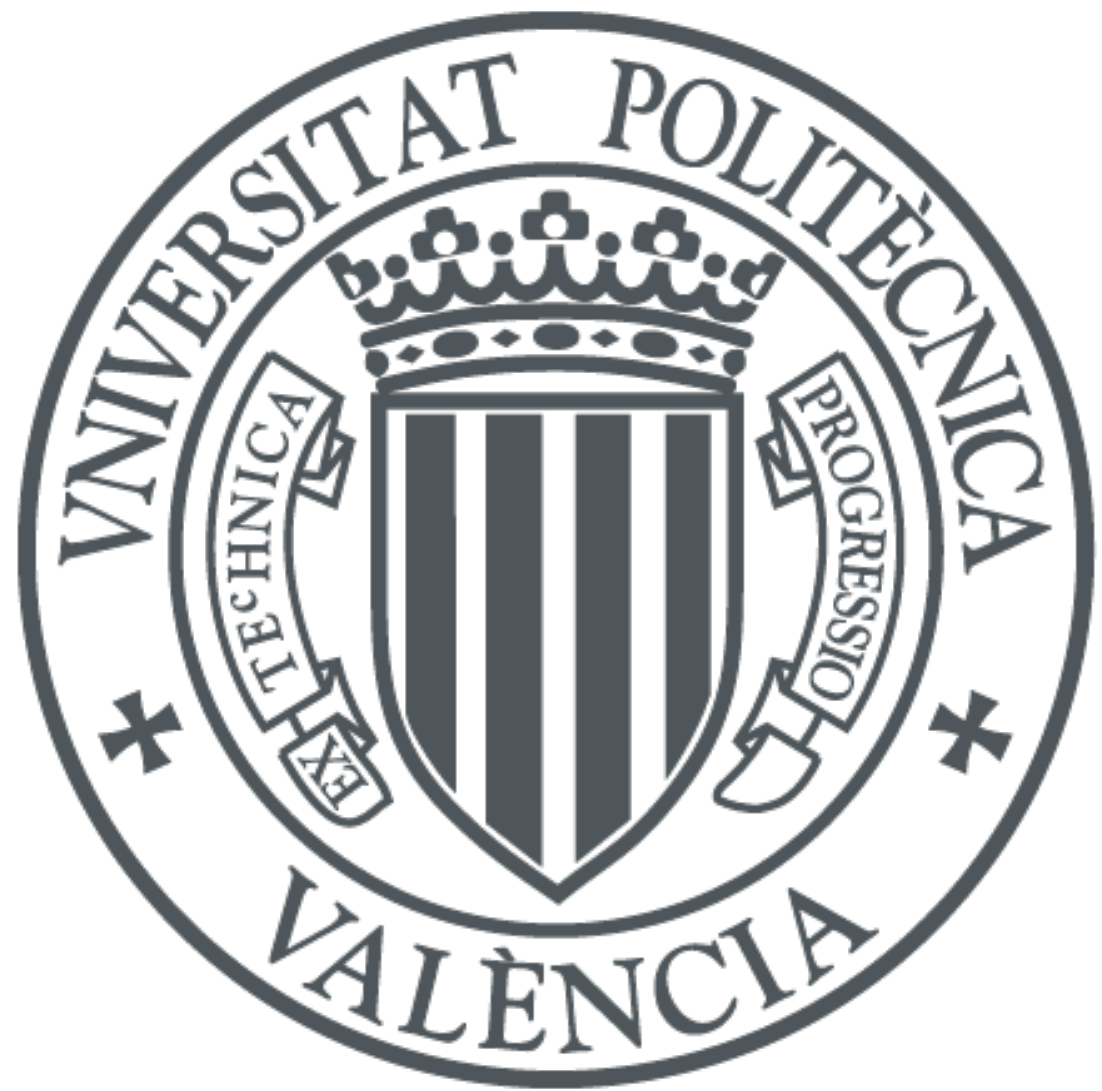

The final publication is available at

http://dx.doi.org/ 10.1109/PIMRC.2018.8580862

Copyright IEEE

Additional Information 


\section{Impact of Receivers Location on the Accuracy of Capsule Endoscope Localization}

\author{
Martina Barbi, Concepcion Garcia-Pardo, \\ Narcís Cardona \\ Institute of Telecommunications and Multimedia \\ Applications (ITeAM) \\ Universitat Politècnica de València \\ 46022, Valencia, Spain \\ marbar6@iteam.upv.es
}

\author{
Andrea Nevarez, Vicente Pons, \\ Matteo Frasson \\ Hospital Universitari i Politècnic, IIS, La Fe, \\ Digestive Diseases Area, \\ 46126 Valencia \\ (email: nevarezja78@gmail.com, \\ pons_vicbel@gva.es,dr.frasson.matteo@gmail.com)
}

\begin{abstract}
In recent years, localization for capsule endoscopy applications using Ultra-Wideband (UWB) technology has become an attractive field of study due to its potential benefits for patients. Performance analysis of RF-based localization techniques are very limited in literature. Most of the available studies rely on software simulations using digital human models. Nonetheless, no realistic studies based on in-vivo measurements has been reported yet. This paper investigates the performance of RSS-based technique for three-dimensional (3D) localization in the UWB frequency band. Impact of receivers selection as well as of the evaluated path loss model on the localization accuracy is investigated. Results obtained through CST-based simulations and from recently conducted in-vivo measurements are presented and compared.
\end{abstract}

Index Terms - Wireless capsule endoscopy (WCE), Ultra-Wideband (UWB), in-body localization, in-vivo measurements, CST simulations

\section{INTRODUCTION}

Wireless Capsule Endoscopy (WCE) is a noninvasive technology which allows physicians to inspect the whole gastrointestinal tract (GI) of the patient. A precise localization of detected diseases is of fundamental importance so the doctor will be able to treat them properly. Beside the fact that current capsule endoscopes provide poor quality images, the position estimation and tracking of the pill through proprietary software supplied to the hospitals is also very inaccurate [1], [2], [3].

Different techniques based on radio frequency signals [4], [5] magnetic fields [6], [7] and image processing algorithms [8], [9] exist in literature to locate the endoscopic capsule. RF-based localization constitutes an efficient and low-cost solution which does not add complexity to the capsule's hardware. Particularly, position estimation using the Received Signal Strength (RSS) is mostly implemented due to its simplicity and less sensitivity to bandwidth limitations
[10], [11] compared to other approaches, such as time of arrival (ToA) [10], [12] or time difference of arrival (TDoA) [12]. Using the RSS, the ranging estimation is performed through a previously established path loss model, which relates the attenuation suffered by the RF signal with the distance from the source. Therefore, the accuracy of the model has a significant impact on the localization performance.

In the latest years, the low part of the UltraWideband (UWB) frequency spectrum $(3.1-5.1 \mathrm{GHz})$ is being under investigation to overcome the limits [13] of the current operating frequency band of capsule endoscopes, (MICS band), such as the poor images quality [1].

Performance analysis of RF-based localization techniques in the UWB frequency band are currently very limited in literature. Specifically, for RSS-based ranging estimation the main issue is the lack of standardized path loss models for in-body to on-body communications at UWB frequencies.

Studies conducted through electromagnetic simulators based on UWB RF signals are presented in [11], [14], [15]. In [16] analysis of ToA-based ranging accuracy are performed through experimental measurements using an homogeneous phantom.

Three main approaches could be used to collect data to be used for the testing of RF-based localization algorithm: software simulations, laboratory measurements and in-vivo experiments [17]. Despite the complexity and the high computational times, software simulations are easy to perform and always a good alternative to obtain data for localization purposes. Laboratory measurements are also a good alternative to collect experimental data but dedicated testbeds and accurate phantom models are required. Regarding invivo measurements, experiments in living animals are not easy to conduct as they are subject to ethical restrictions and also requires dedicated facilities and a specialized medical equipment. However, this is the 
most realistic scenario where developed localization algorithms can be tested compared to simulations (ideal environment) and laboratory measurements (controlled environment).

In this paper, performance of RSS-based localization technique is investigated in the 3.1-5.1 GHz UWB frequency band. The impact of receivers selection and of the path loss model used for ranging estimation is analyzed through CST-based simulations and measurements data obtained from a recently conducted in-vivo experiment. To the best of the author's knowledge no studies of RF-based localization techniques at UWB frequencies, through in-vivo experiments has been reported yet.

The remainder of the paper is organized as follows: Section II describes the CST-based simulation setup as well as the conducted in-vivo measurements. Section III briefly presents the implemented RSS-based localization technique. Performance metrics along with the obtained results are presented in Section IV. Finally conclusions and future research plans are discussed in Section V.

\section{SIMULATIONS AND MEASUREMENT SETUP}

\section{A. CST-based simulations}

Simulations using the commercial software $\mathrm{CST}^{\circledR}$ $\mathrm{MWS}^{\circledR}$, were conducted in the 3.1-5.1 GHz UWB frequency band. The S-parameter was evaluated by the software through the Finite Difference Time Domain (FDTD) method. In order to compare the simulation results with the in-vivo ones, the abdominal part of a human female CAD model (Nelly) was used. This model mimics the permittivity of the human tissues also involved in the in-vivo experiment i.e. skin, fat and muscle, which has permittivity similar to the small bowel. No internal organs as well as no blood flow were considered, in order to reduce the computational time of the simulations. Figure 1 shows the described model along with the in-body and on-body antenna positions considered in this study.

The antennas used in the simulations are UWB omnidirectional patch antennas, specifically designed to operate inside and on the surface of the human body [18], [19].

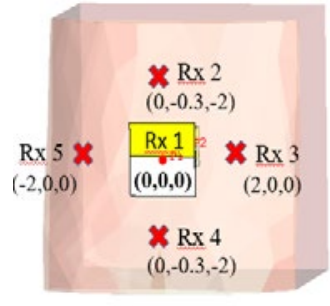

(a)

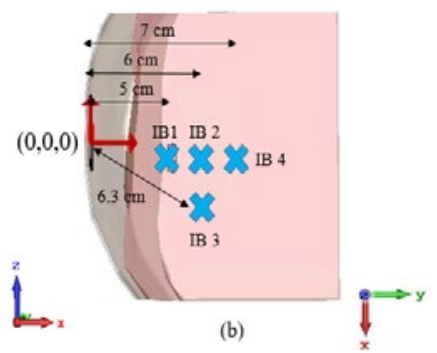

Fig 1. On-body (a) and in-body (b) antenna locations (top view)

Measurements were performed by moving the in- body antenna in steps of $1 \mathrm{~cm}$ in four different locations along $x$ and $y$ axis inside the muscle layer (Fig. 1(b)). Five on-body antenna positions, with a separation of 2 $\mathrm{cm}$ along $\mathrm{x}$ and $\mathrm{z}$ axis and of $0.3 \mathrm{~cm}$ along $\mathrm{y}$ axis, were considered on the abdominal region of the CAD model (Fig. 1(a)). As shown in Fig. 1, the center (P1) of the onbody receiving antenna $\mathrm{Rx} 1$ is taken as the origin of the reference system used to evaluate the real and estimated positions of the in-body antenna.

\section{B. In-vivo measurements}

In-vivo measurements were conducted in a living porcine model, at the Hospital Universitari i Politècnic la $\mathrm{Fe}$ in Valencia, Spain. A brief description of the experiment is given in this section, in order to highlight the main aspects. Further and more detailed informations can be found in [20].

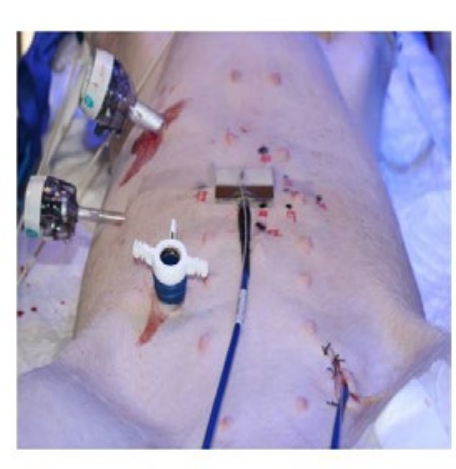

(a)

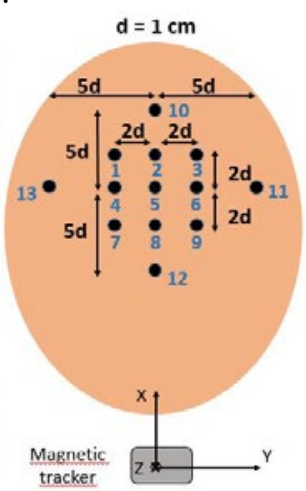

(b)
Fig 2. In-vivo measurements for one on-body position (a) and grid of all on-body position (top view) (b) [21]

Same antennas described in Section II.A were used for the experiment. The $S_{21}$ parameter was measured for each in-body-on-body antenna position by means of a Vector Network Analyzer (VNA). A magnetic tracker equipped with two magnetic sensors, attached to the inbody and on-body antenna, was used to evaluate the real and estimated distances between them as well as their cartesian coordinates with respect to the magnetic transmitter's reference system (Fig. 2(b)). The in-body antenna was moved, through laparoscopy, in two different locations of the abdomen. For each location, the on-body antenna was placed manually in thirteen different positions on the abdomen of the porcine model (Fig. 2(b)), in direct contact with the skin (Fig. 2(a)).

Measurements were taken in the 3-6 GHz UWB frequency band, considering 1601 resolution points in frequency. For each pair of in-body to on-body position five snapshots of the channel $\left(\mathrm{S}_{21}\right)$ were taken. Only values above the noise level $(-90 \mathrm{dBm})$ were taken into account. Antenna separation distance and antenna coordinates were evaluated 100 times per snapshot by the magnetic tracker. Finally, averaging the collected measurements, $\mathrm{S}_{21}$, antennas separation distance and antennas coordinates were calculated. 


\section{RSS-BASED LOCALIZATION ALGORITHM}

For the in-vivo measurements campaign data from 3.1 to $5.1 \mathrm{GHz}$ were considered because for antenna distances above 8-9 cm measurements are below the noise level [22]. So, for CST-based simulations same distance range between antennas (up to $8 \mathrm{~cm}$ ) is considered in order to compare the obtained results with the in-vivo ones.

For both cases, simulations and in-vivo measurements, same 3D localization algorithm based on the Received Signal Strength is applied.

Ranging distance estimation is performed as in [23] by using the log-distance path loss model evaluated from the measured $S_{21}$, for each in-body to on-body antenna position. Then, the coordinates of the in-body antenna are estimated using the Non Linear Least Square method described in [24]. The sum of the square errors is minimized through the Levenberg-Marquardt algorithm [25]. The receivers selection criteria as well as the metrics used to evaluate the localization performance are explained in Section IV.

\section{LOCALIZATION RESULTS}

\section{A. Performance Metrics}

In order to assess the goodness of the obtained localization results, the localization error, $L E$, for $3 \mathrm{D}$ positioning and its relative error can be defined as:

$$
\begin{gathered}
L E=\sqrt{\left(x_{I B}-x_{I B_{-} e s t}\right)^{2}+\left(y_{I B}-y_{I B_{-}} e s t\right)^{2}+\left(z_{I B}-z_{I B} \text { est }\right)^{2}} \\
\operatorname{Rel} L E=\frac{L E}{\sqrt{x_{I B}^{2}+y_{I B}^{2}+z_{I B}^{2}}}
\end{gathered}
$$

where $\left(x_{I B}, y_{I B}, z_{I B}\right)$ and $\left(x_{I B \_e s t}, y_{I B_{-} e s t}, z_{I B} \_\right.$est $)$are the real and estimated coordinates of the in-body antenna, respectively.

\section{B. Results from CST simulations}

For the CST-based simulations path loss parameters of the log-distance fitting model $\mathrm{PL}_{0, \mathrm{dref}}=-15.83$ and $\mathrm{n}=10.52$ were obtained, considering a reference distance of $\mathrm{d}_{0}=1 \mathrm{~cm}$. The model along with the measured data is depicted in Fig. 5.

In order to estimate the $\left(\mathrm{x}_{\mathrm{IB}}, \mathrm{y}_{\mathrm{IB}}, \mathrm{z}_{\mathrm{IB}}\right)$ coordinates of the in-body antenna, the method described in Section III is applied using different combinations of four receivers. Figure 3 shows the relative localization error obtained for the four simulated in-body positions (located at 5, 6, 6.3 and $7 \mathrm{~cm}$ with respect to the origin of the reference system (Fig. 1(b))) for five different combinations of four receivers.

Results in Fig. 3 show that for the first three in-body positions the lowest error is obtained with the combination of receivers $1,2,3,5$. These receivers for all four in-body locations, are experiencing the lowest path loss, i.e. the highest received power.

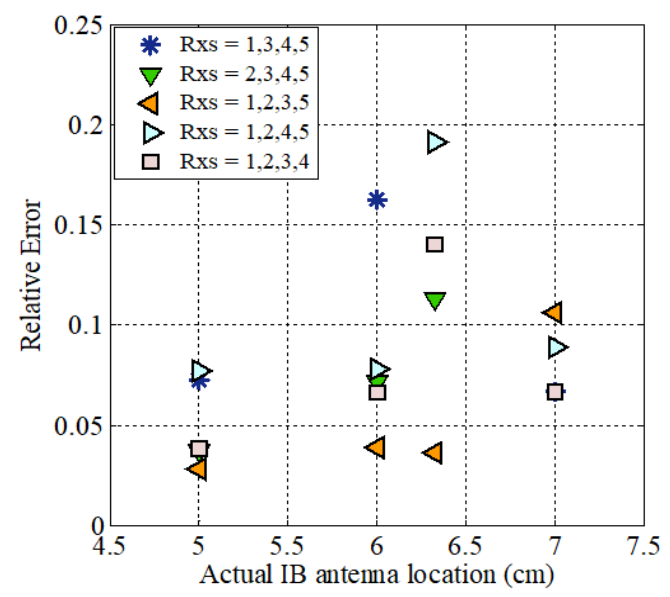

Fig 3. Relative localization error vs actual in-body antenna location for different combinations of four receivers

This can be observed in the histogram in Fig. 4 which depicts the average path loss experienced by each combination of four receivers, represented by a different colorbar, for the four in-body locations considered. For the combination $1,2,3,5$, the orange bar, the average experienced path loss is the lowest compared to the other combination of receivers. Therefore, it is reasonable to expect higher positioning accuracy, i.e. lower localization errors, using these four receivers rather than other combinations. However, this is true, only for three in-body positions. For the in-body location at $7 \mathrm{~cm}$, as shown in Fig. 3, the lowest error is achieved by the combination of receivers $1,2,3,4$. This can be explained by looking, in Fig. 5, at the dispersion of the path loss values, corresponding to these two combinations, with respect to the fitting model. Path loss values related to receivers 1, 2, 3, 4 (light pink squares) are slightly closer to the fitting model curve with respect to those related to receivers $1,2,3,5$ (orange triangles), leading to lower ranging errors (difference between real antennas distance and the estimated one) in the estimation of the antennas distance.

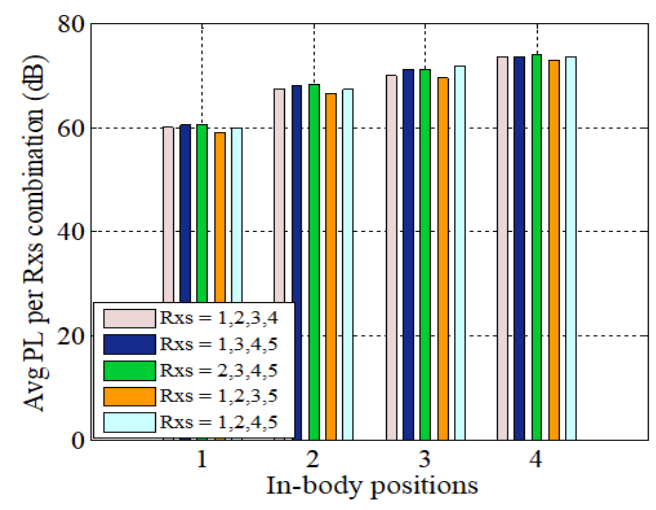

Fig 4. Histogram of average path loss per in-body position for different combinations of receivers 
In fact, the average ranging error passes from $0.1672 \mathrm{~cm}$ for the combination $1,2,3,5$ to $0.1270 \mathrm{~cm}$ for the combination $1,2,3,4$. This, in turn, affects the accuracy of the minimization error algorithm used for the estimation of the coordinates, resulting in a lower localization error, as shown in Fig. 3.

In order to validate these preliminary results obtained for an ideal environment (software simulations) in a more realistic scenario, same analysis is conducted in the next section for the in-vivo measurements case.

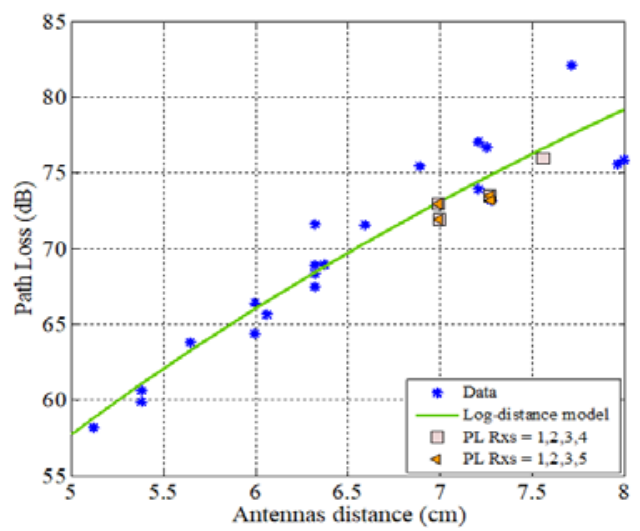

Fig 5. Simulated path loss values and fitting model along with path loss values of selected receivers for in-body position at $7 \mathrm{~cm}$

\section{Results from in-vivo measurements}

Regarding the in-vivo measurements path loss parameters of the log-distance fitting model $P L_{0, \text { dref }}=21.84$ and $n=5.44$ were obtained, considering a reference distance of $d_{0}=1 \mathrm{~cm}$. The model along with the measured data is presented in Fig. 7.

As for the CST-based simulations the estimation of the in-body antenna coordinates $\left(\mathrm{x}_{\mathrm{IB}}, \mathrm{y}_{\mathrm{IB}}, \mathrm{z}_{\mathrm{IB}}\right)$ is performed by selecting the receivers experiencing the lowest path loss. Particularly, performance is investigated when increasing the number of receivers used for the localization, as during this experiment more on-body positions compared to the simulated ones were measured. Figure 6 shows the relative localization error values obtained increasing the number of receivers experiencing the highest received power (lowest path loss), starting with 4 and then up to 13 , for the two inbody positions under study.

Results show that increasing the number of receivers the localization accuracy does not always increase as it would be expected. Looking at Fig. 6(a) for in-body position 1, passing from 4 to 6 receivers, for example, the localization error slightly decreases. However, this does not happen increasing the receivers from 6 to 8 or from 9 to 11. The same behavior can be observed in Fig. 6(b) for in-body position 2, passing from 6 receivers, where the error is the lowest, to 13 receivers, where the error slightly increases. This can be explained, as for the CST-based simulations, looking at the dispersion of the path loss values related to the selected receivers, with respect to the fitting model. Figure 7 depicts, as an example, the path loss values related to the six and seven receivers selected according to the lowest path loss criteria for in-body position 2 .

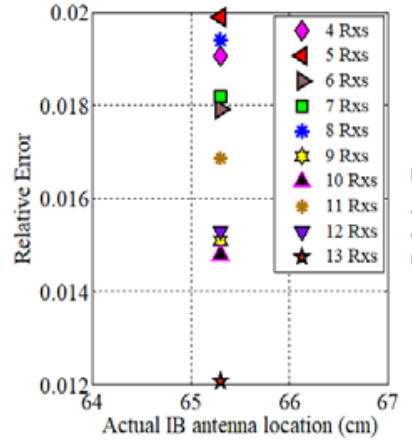

(a)

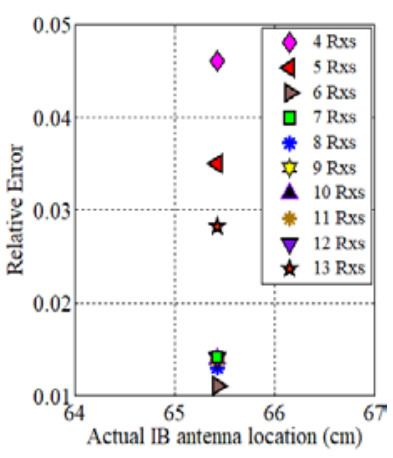

(b)
Fig 6. Relative localization error vs actual in-body antenna location for in-body position 1 (a) and in-body position 2 (b)

Using seven receivers (green squares) instead of six (brown triangles) the average ranging error increases as the seventh selected receiver has a mean path loss value far away from the fitting curve (green square around 4 $\mathrm{cm})$, which means higher inaccuracy in the antennas distance estimation. In fact, the average ranging error passes from $0.69 \mathrm{~cm}$ with six receivers to $0.86 \mathrm{~cm}$ with seven receivers. Same behavior, i.e. an increment of the average ranging error, has been observed using more than seven receivers, up to thirteen, where the average ranging error reaches $1.09 \mathrm{~cm}$. This affects the accuracy of the minimization error algorithm resulting in a slightly higher localization error, as shown in Fig. 6(b). In conclusion, increasing the number of receivers experiencing the highest power not always improves the localization performance, as the dispersion of the selected mean path loss values with respect to the fitting model also affects the accuracy of the algorithm.

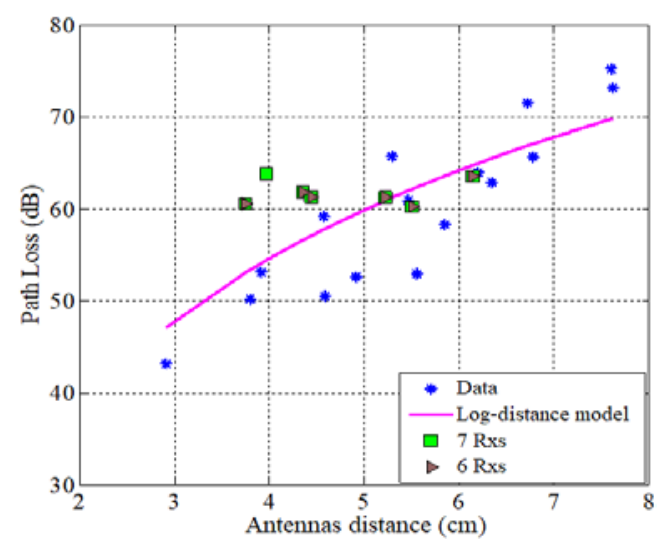

Fig. 7. Measured path loss values and fitting model along with path loss values of selected receivers for in-body position 2

\section{CONCLUSIONS}

In this paper, performance of RSS-based localization technique for in-body to on-body applications, in the 3.1 
to $5.1 \mathrm{GHz}$ frequency band have been investigated. Three dimensional (3D) localization is performed through CST-based simulations and in-vivo measurements. Results show that the selection of receivers experiencing the highest power as well as increasing the number of receivers, used for 3D positioning, not always leads to the best localization precision. This very much depends on the accuracy of the evaluated path loss model. For selected path loss values far from the fitting model the ranging error increases, resulting in higher localization errors.

Future research plans involve more extensive measurement campaigns and simulations in order to derive an accurate and more general path loss model. Moreover, performance analysis on combinations of RSS-based approach with other localization technique (image-based, for example) or other algorithms (Neural Networks, for example) will be investigated in order to improve the positioning accuracy.

\section{ACKNOWLEDGMENT}

This work was supported by the European Union's H2020:MSCA:ITN program for the "Wireless In-body Environment Communication- WiBEC" project under the grant agreement no. 675353. This work was also funded by the Ministerio de Economía y Competitividad, Spain (TEC2014-60258-C2-1-R), by the European FEDER funds.

\section{REFERENCES}

[1] G. Ciuti, A. Menciassi, and P. Dario, "Capsule Endoscopy: From Current Achievments to Open Challenges," IEEE Trans. Biomed. Eng., vol. 4, pp. 59-72, 2011.

[2] M. F. Hale, R. Sidhu, and M. E. McAlindon, "Capsule endoscopy: Current practice and future directions," World J. Gastroenterol., vol. 20, no. 24, pp. 7752-7759, 2014.

[3] "Given Imaging." [Online]. Available: http://www.givenimaging.com/en-int/InnovativeSolutions/Product-Support/pillcam-help-center/Software Instructional Videos/Pages/default.aspx.

[4] K. Pahlavan et al., "RF localization for wireless video capsule endoscopy," Int. J. Wirel. Inf. Networks, vol. 19, no. 4, pp. 326-340, 2012

[5] M. Pourhomayoun, M. Fowler, and Z. Jin, "A novel method for medical implant in-body localization," in Proceedings of the Annual International Conference of the IEEE Engineering in Medicine and Biology Society, EMBS, 2012, pp. 5757-5760.

[6] C. Hu, M. Q. Meng, and M. Mandal, "Efficient Magnetic Localization and Orientation Technique for Capsule Endoscopy," in IEEE/RSJ International Conference on Intelligent Robots and Systems, 2005, pp. 3365-3370.

[7] R. Kuth, J. Reinschke, and R. Rockelein, "Method for determining the position and orientation of an endoscopy capsule guided through an examination object by using a navigating magnetic field generated by means of a navigation device," US20070038063, 2007.

[8] G. Bao, L. Mi, and K. Pahlavan, "A Video Aided RF Localization Technique for the Wireless Capsule Endoscope (WCE) inside Small Intestine," in Proceedings of the 8th International Conference on Body Area Networks, 2013, no. February 2016, pp. 55-61.

[9] G. Bao and K. Pahlavai, "Motion estimation of the endoscopy capsule using region-based Kernel SVM classifier," in IEEE
International Conference on Electro Information Technology, 2013.

[10] U. I. Khan, K. Pahlavan, and S. Makarov, "Comparison of TOA and RSS based techniques for RF localization inside human tissue," in Proceedings of the Annual International Conference of the IEEE Engineering in Medicine and Biology Society, EMBS, 2011, pp. 5602-5607.

[11] A. S. Bjørnevik, "Localization and Tracking of Intestinal Paths for Wireless Capsule Endoscopy," M. S. Thesis, DET, Norwegian University of Science and Technology, 2015.

[12] A. R. Nafchi, S. T. Goh, and S. A. R. Zekavat, "Circular arrays and inertial measurement unit for DOA/TOA/TDOA-based endoscopy capsule localization: Performance and complexity investigation," IEEE Sens. J., vol. 14, no. 11, pp. 3791-3799, 2014.

[13] Q. W. Jianqing Wang, Body Area Communications: Channel Modeling, Communication Systems, and EMC. 2013.

[14] B. Moussakhani, J. T. Flåm, S. Støa, I. Balasingham, and T. Ramstad, "On localisation accuracy inside the human abdomen region," IET Wirel. Sens. Syst., vol. 2, no. 1, pp. 9-15, Mar. 2012.

[15] M. Kanaan and M. Suveren, "In-body ranging with ultrawideband signals: Techniques and modeling of the ranging error," Wirel. Commun. Mob. Comput., vol. 2017, 2017.

[16] Y. Ye, "Bounds on Rf Cooperative Localization for Video Capsule Endoscopy," Ph.D. dissertation, ECE, WPI, Worcester, MA, USA, 2013.

[17] C. Garcia-Pardo, C. Andreu, A. Fornes-Leal, S. CastelloPalacios, S. Perez-Simbor, M. Barbi, A. Valles-Lluch, "Ultrawideband Technology for Medical In-Body Sensor Networks: An Overview of the Human Body as a Propagation Medium, Phantoms, and Approaches for Propagation Analysis," IEEE Antennas and Propagation Magazine, 2018.

[18] C. Andreu, C. Garcia-Pardo, A. Fornes-Leal, M. CabedoFabrés, and N. Cardona, "UWB In-Body Channel Performance by Using a Direct Antenna Designing Procedure," in 11th European Conference on Antennas and Propagation (EUCAP), 2017, p. 5.

[19] C. Tarín, P. Martí, L. Traver, N. Cardona, J. A. Díaz, and E. Antonino, "UWB channel measurements for hand-portable devices: A comparative study," in IEEE International Symposium on Personal, Indoor and Mobile Radio Communications, PIMRC, 2007.

[20] S. Perez-Simbor, C. Garcia-Pardo, C. Andreu, M. Frasson, N. Cardona, "Path Loss Models for Implanted Devices in the Gastrointestinal Area in the UWB Frequency Band," IEEE Trans. Antennas Propag., Special Issue on Wireless Healthcare Biotechnology, 2018, p. under review.

[21] M. Barbi, C. Garcia-Pardo, A. Nevarez, V. Pons, N. Cardona, "UWB RSS-based Localization for Capsule Endoscopy using a Multilayer Phantom and In-Vivo Measurements," IEEE Trans. Antennas Propag., Special Issue on Wireless Healthcare Biotechnology, 2018, p. under review.

[22] S. Perez-Simbor, M. Barbi, C. Garcia-Pardo, "Initial UWB inbody channel characterization using a novel multilayer phantom measurement setup," in IEEE Wireless Communications and Networking Conference, WCNC, 2018, p. accepted for publication.

[23] M. Barbi, S. Perez-Simbor, C. Garcia-Pardo, C. Andreu, N. Cardona, "Localization for Capsule Endoscopy at UWB Frequencies using an Experimental Multilayer Phantom," in IEEE Wireless Communications and Networking Conference, $W C N C, 2018$, p. accepted for publication.

[24] W. Murphy and W. Hereman, "Determination of a position in three dimensions using trilateration and approximate distances," Department of Mathematical and Computer Sciences, Colorado School of Mines, Golden, Colorado, MCS95, vol. 7. p. 19, 1995.

[25] Charles L. Lawson and Richard J. Hanson, Solving Least Squares Problems. 1995. 